\title{
Silica promotes the transdifferentiation of rat circulating fibrocytes in vitro
}

\author{
WU YAO, SUNA LIU, JU LI and CHANGFU HAO \\ Department of Public Health, Zhengzhou University, Zhengzhou, Henan 450001, P.R. China
}

Received October 22, 2014; Accepted July 21, 2015

DOI: $10.3892 / \mathrm{mmr} .2015 .4212$

\begin{abstract}
To investigate the effects of silica on circulating fibrocytes (cFbs), the present study established a primary culture model of rat alveolar macrophages and cFbs in vitro. Macrophages were treated with free silica, and their supernatant was used to stimulate cFbs. The mRNA expression levels of collagen I, collagen III and $\alpha$-smooth muscle actin (SMA) in $\mathrm{cFbs}$ were analyzed by reverse transcription-quantitative polymerase chain reaction. The intracellular and extracellular protein expression levels of collagen I, collagen III and $\alpha$-SMA were detected by ELISA and immunofluorescence staining. The results indicated that in the cell model, the free silica effectively increased the protein and mRNA expression levels of collagen-I, collagen-III and $\alpha$-SMA. The free silica significantly promoted the transdifferentiation of cFbs into myofibroblasts in a dose-and time-dependent manner.
\end{abstract}

\section{Introduction}

Silicosis, which is among the longest known occupational diseases, is caused by the inhalation of silica particles, usually at low levels but over long time periods, which eventually causes irreversible lung fibrosis. Regardless of its source, purity and age, crystalline silica induces various pathological reactions in the lung and causes irreversible fibrosis and damage $(1,2)$. Fibrocytes are a cell type arising from monocyte precursors expressing surface markers for leukocytes and mesenchymal cells, which are capable of differentiating into myofibroblasts (3-5). They were described in 1994 as a circulating, bone marrow-derived cells with the ability to adopt a mesenchymal phenotype (4). Fibrocytes are initially present in injured organs and have the inflammatory features of macrophages; they have been shown to participate in granuloma formation, antigen presentation and various fibrotic disorders (6).

Correspondence to: Professor Changfu Hao, Department of Public Health, Zhengzhou University, 100 Kexue Road, Zhengzhou, Henan 450001, P.R. China

E-mail: haochangfu@126.com

Key words: circulating fibrocytes, free silica dust, collagen I, collagen III, $\alpha$-smooth muscle actin
Fibrocytes represent a minor component of the circulating pool of leukocytes and express a characteristic pattern of markers, including collagen I and collagen III (4). $\alpha$ smooth muscle actin ( $\alpha$-SMA) expressed on the surface of fibrocytes is a surface marker of myofibroblasts $(7,8)$. A previous study demonstrated that peripheral blood fibrocytes migrate to skin wound chambers in humans as well as in mice (9). Fibrocytes have been identified at sites of active fibrosis and in fibrotic pathologies, including hypertrophic scars, asthma and idiopathic pulmonary fibrosis (IPF) (10-12). Fibrocytes contribute to fibrogenesis by directly producing collagen, hematopoietic growth factors, inflammatory cytokines and chemokines (13).

It is well known that fibroblasts are responsible for repair and remodeling within the lung. Circulating fibrocytes (cFbs) in the blood are involved in tissue damage repair and were shown to be able to transform into fibrocytes in vitro (5). cFbs have been described as a potential source of increased extracellular matrix and myofibroblasts, markers of fibrotic pathologies, and to contribute to the pathogenesis of pulmonary fibrosis (14). Without treatment, continued fibrosis leads to loss of lung function and ultimately, sufferers succumb to the disease. cFbs locate to the damaged area of lung tissue via the circulatory system through the action of chemotactic factors and differentiate into active fibrocytes prior to repairing and rebuilding the lung tissue $(15,16)$. cFbs may function as precursors of myofibroblasts in proliferative vitreoretinopathy membranes (8). cFbs have an important role in fibrosis damage, including pneumoconiosis (17).

The effects of crystalline silica $\left(\mathrm{SiO}_{2}\right)$ on cFbs and on the development of silicosis have yet to be determined. cFbs may be recruited to injured lungs as an integral component of the pathogenesis of pulmonary fibrosis. The aim of the present study was to evaluate the effects of free $\mathrm{SiO}_{2}$ on the differentiation of cFbs in vitro, specifically with regard to the effects of free $\mathrm{SiO}_{2}$ on the expression levels of collagen I, collagen III and $\alpha$-SMA. Sprague Dawley (SD) rat $\mathrm{cFbs}$ and alveolar macrophages (AMs) were separated and cultured in order to establish a primary rat culture model of $\mathrm{AMs}$ and cFbs in vitro. AMs were treated with free $\mathrm{SiO}_{2}$ at 0, 20, 40, 60, 80, 100 and $120 \mu \mathrm{g} / \mathrm{ml}$, the cell culture supernatant was collected and then used to stimulate $\mathrm{cFbs}$. $\mathrm{cFbs}$ stimulated by the supernatant were then identified by immunohistochemical analysis. The mRNA expression levels of collagen I, collagen III and $\alpha$-SMA in the cFbs were analyzed by reverse transcription-quantitative polymerase chain reaction (RT-qPCR). The extracellular protein 
expression levels of collagen I, collagen III and $\alpha$-SMA were detected by ELISA. The results of the present study further clarified the role of $\mathrm{cFbs}$ in silicosis.

\section{Materials and methods}

Animals. Specific pathogen-free male SD rats were purchased from the animal center of Henan province (Henan, China). The rats (age, 8-13 weeks; weight, 120-150 g) were housed in polycarbonate isolator cages with autoclaved bedding, and provided with ad libitum access to autoclaved reverse-osmosis water and standard rat food. Animals were maintained in accordance with the guidelines of the Chinese Association of Laboratory Animal Care, all experiments and surgical procedures complied with the relevant provisions of the Experimental Animal Ethics Committee of Zhengzhou University (Zhengzhou, China).

Reagents. The $\mathrm{SiO}_{2}$ dust (purity, $>99 \%$; particle size, $<5 \mu \mathrm{m}$ ) was obtained from The Center for Disease Control and Prevention (Beijing, China) and sterilized by hot air sterilization at $190^{\circ} \mathrm{C}$ for $1.5 \mathrm{~h}$. The stock solutions $(100 \mathrm{mg} / \mathrm{ml})$ of $\mathrm{SiO}_{2}$ were suspended in Dulbecco's modified Eagle's medium (DMEM; Beijing Solarbio Science \& Technology Co., Ltd., Beijing, China) and diluted to the desired working concentrations for further experimentation.

Cell isolation and culture. The cFbs were prepared as previously described (4). Total peripheral blood lymphocytes were first isolated from $45 \mathrm{ml}$ rat blood by centrifugation in Ficoll ${ }^{\circledR}$ Paque Plus (GE Healthcare Life Sciences, Little Chalfont, UK) according to the manufacturer's instructions. Following overnight culture in six-well plates in high-glucose DMEM supplemented with $20 \%$ heat-inactivated fetal bovine serum (FBS; Hyclone, Logan, UT, USA), non-adherent cells were removed by gentle aspiration. The experiments were conducted at $37^{\circ} \mathrm{C}$ in a $5 \% \mathrm{CO}_{2}$ humidified atmosphere after six weeks of culture.

Rat AMs were collected from the SD rats by bronchoalveolar lavage. Briefly, the rats were anesthetized with $2 \%$ pentobarbital (Sigma-Aldrich, Shanghai, China) by intraperitoneal injection, and following tracheal exposure and cannulation, the airways of the rats were gently treated with $5 \mathrm{ml}$ cold sterile D-Hank's solution. This procedure was repeated two additional times. The bronchoalveolar lavage fluid was collected and centrifuged at $800 \mathrm{x} \mathrm{g}$, for $5 \mathrm{~min}$ at $4^{\circ} \mathrm{C}$ to form pellets of AMs, which were re-suspended in DMEM supplemented with $10 \%$ FBS. The AMs were counted using a hemocytometer, and the cells were incubated at $37^{\circ} \mathrm{C}$ for $2 \mathrm{~h}$ in an atmosphere containing $5 \% \mathrm{CO}_{2}$. The medium was then discarded and any non-adherent cells were removed by washing.

Fluorescence-activated cell sorting analysis. The cells were harvested after six weeks of culture with $0.25 \%$ trypsin (Beijing Solarbio Science \& Technology Co., Ltd.) and pelleted by centrifugation for $7 \mathrm{~min}$ at $250 \mathrm{x} \mathrm{g}$. The cells were then re-suspended in $0.5 \mathrm{ml}$ phosphate-buffered saline (PBS), and phycoerythrin-conjugated anti-collagen I and fluorescein isothiocyanate-conjugated anti-CD45 antibodies
(BD Biosciences, Franklin Lakes, NJ, USA) were added according to the manufacturer's instructions. Following incubation in a dark environment for $30 \mathrm{~min}$ at $4^{\circ} \mathrm{C}$, cell fluorescence was evaluated by flow cytometry (Accuri C6; BD Biosciences).

Cytotoxicity assay and cellular activity. The AMs were seeded into 96 -well plates at a density of $3 \times 10^{5}$ cells/well ( $250 \mu \mathrm{l}$ media per well). After $24 \mathrm{~h}$ of incubation, the media was replaced with fresh media supplemented with $\mathrm{SiO}_{2}$ at 0, 20, 40, 60, 80,100 or $120 \mu \mathrm{g} / \mathrm{ml}$. Each concentration was assayed in six replicates, and the control wells received no $\mathrm{SiO}_{2}(0 \mu \mathrm{g} / \mathrm{ml})$. An MTT assay was performed after $24 \mathrm{~h}$ of culture. cFbs from six wells in each group were seeded in 96-well plates at a density of $1.5 \times 10^{4}$ cells/well $(200 \mu \mathrm{l}$ media per well). A total of $24 \mathrm{~h}$ after seeding, the cells were collected and counted under a light microscope (Eclipse TS100-F; Nikon, Tokyo, Japan) with trypan blue exclusion (Sigma-Aldrich). The MTT assay was performed once daily from the first day of culture, the cells from six wells of each day were assessed for ten consecutive days, and the cell numbers were used to produce a growth curve. The optical density (OD) was measured at $490 \mathrm{~nm}$ using a plate microreader (Tecan Infinite M200; Tecan, Wetzlar, Germany). The proliferation rate was determined using the following formula: Cell proliferation $(\%)=\mathrm{OD}_{\text {experimental }}$ samples $/ O D_{\text {control }} \times 100 \%[n=6$, mean \pm standard deviation (SD)]. The cell growth curve was used to determine the activity levels of the cFbs, and to evaluate the inhibitory effects of $\mathrm{SiO}_{2}$ on the growth of the macrophages in order to ascertain the maximum non-lethal concentration of $\mathrm{SiO}_{2}$.

Cell treatment. A primary culture model of rat AMs and cFbs was established in vitro. After $24 \mathrm{~h}$ of culture, the AMs were treated with various concentrations of $\mathrm{SiO}_{2}(0,20,40$, $60,80,100$ and $120 \mu \mathrm{g} / \mathrm{ml})$. After $24 \mathrm{~h}$ of $\mathrm{SiO}_{2}$ treatment, the cell culture supernatant and cells were collected for the subsequent experiments. After three days of cFbs culture, the cells were treated with supernatant for $24 \mathrm{~h}$. The supernatant was collected from the AMs, which were stimulated with $\mathrm{SiO}_{2}$ at various concentrations $(0,20,40,60$ and $80 \mu \mathrm{g} / \mathrm{ml})$, and each concentration was assayed in six replicates. The cell culture supernatant was collected from AMs and was then transferred to $\mathrm{cFb}$ each day for 28 days consecutively, the cFbs were cultured with DMEM supplemented with $10 \%$ FBS (control), $0 \mu \mathrm{g} / \mathrm{ml} \mathrm{SiO}_{2}$-treated AM supernatant $(0 \mu \mathrm{g} / \mathrm{ml}, 2 \mathrm{ml}$ for $1 \times 10^{5}$ cells) and $80 \mu \mathrm{g} / \mathrm{ml} \mathrm{SiO}_{2}$-treated AM supernatant (80 $\mu \mathrm{g} / \mathrm{ml}, 2 \mathrm{ml}$ for $1 \times 10^{5}$ cells) respectively. After cultivation for 3, 6, 9, 12, 15 and 18 days for collagen I and Collagen III detection, and 13, 16, 19, 22, 25 and 28 days for $\alpha$-SMA detection, the cell culture supernatant and cells were collected for the subsequent experiments.

Immunohistochemistry. Immunofluorescence staining was used to determine the levels of collagen I, collagen III and $\alpha$-SMA proteins in the $\mathrm{cFbs}$. The $\mathrm{cFbs}$ were fixed in $4 \%(\mathrm{v} / \mathrm{v})$ paraformaldehyde (Sigma-Aldrich) for $20 \mathrm{~min}$ at $4^{\circ} \mathrm{C}$. To suppress endogenous peroxidase activity, the samples were treated with $3 \%$ hydrogen peroxide solution (Wuhan Boster Biological Technology, Ltd., Wuhan, China) for $15 \mathrm{~min}$ and rinsed with PBS. To prevent non-specific immune reactions, 
Table I. Primers for $\beta$-actin, collagen I, collagen III and $\alpha$-SMA mRNA and their annealing temperatures.

\begin{tabular}{llcc}
\hline Gene & \multicolumn{1}{c}{ Primers } & $\begin{array}{c}\text { Predicted } \\
\text { size }(\mathrm{bp})\end{array}$ & $\begin{array}{c}\text { Annealing } \\
\text { temperature }\left({ }^{\circ} \mathrm{C}\right)\end{array}$ \\
\hline$\beta$-actin & F, 5'-CCCATCTATGAGGGTTACGCT-3' & 519 & 54 \\
Rollagen I & R, 5'-TTTAATGTCACGCACGATTTC-3' & & 51 \\
& F, 5'-CCCACCCCAGCCGCAAAGAT-3' & 352 & 52 \\
Collagen III & R, 5'-TTGGGTCCCTCGACTCCTACA-3' & & 51 \\
\multirow{2}{*}{$\alpha$-SMA } & F, 5'-TGCCCACAGCCTTCTACACCT-3' & 240 & 51 \\
& R, 5'-CAGCCATTCCTCCCACTCCAG-3' & & 5121 \\
& F, 5'-CCGAGATCTCACCGACTACC-3' & & 5 \\
\hline
\end{tabular}

F, forward; R, reverse.

the samples were treated with $3 \%$ normal horse serum (Wuhan Boster Biological Technology, Ltd.) for 20 min. Non-specific staining was prevented by omitting primary antibodies and rat non-immune serum. The slides were gently agitated and then incubated with mouse anti-rat $\alpha$-SMA monoclonal antibody (dilution 1:50; cat. no. BM0002; Wuhan Boster Biological Technology, Ltd.), mouse anti-rat collagen I polyclonal antibody (dilution, 1:50; cat. no. BA0325; Wuhan Boster Biological Technology, Ltd.) and mouse anti-rat collagen III polyclonal antibody (dilution, 1:50; cat. no. BA0326; Wuhan Boster Biological Technology, Ltd.) at $37^{\circ} \mathrm{C}$ for $90 \mathrm{~min}$. Following washing with PBS, horseradish peroxidase-conjugated goat-anti-mouse monoclonal antibody (dilution, 1:200; OriGene Technologies, Inc., Beijing, China) was added and the solution was incubated at $37^{\circ} \mathrm{C}$ for $30 \mathrm{~min}$, followed by rinsing with PBS. Subsequently, avidin-biotin complex solution was added to the solution and incubated for $30 \mathrm{~min}$. The slides were rinsed with PBS, stained with 3-3'-diaminobenzidene (Origene Technologies, Inc.) for $5 \mathrm{~min}$ and rinsed with the buffer solution, as previously described $(18,19)$.

Extraction and RT-qPCR analysis. RNA extraction and RT-qPCR analysis of the mRNA expression levels of collagen I, collagen III and $\alpha$-SMA were performed as previously described (20). The primers were designed and synthesized by Takara Biotechnology Co., Ltd. (Dalian, China) and the annealing temperatures used for the PCR are shown in Table I. A PrimerScript RT reagent kit (Tiangen Biotech Co., Ltd., Beijing, China) was used to reverse-transcribe the total RNA according to the manufacturer's instructions. The resulting cDNA was sequentially amplified with 2X PCR Master Mix (Tiangen Biotech Co., Ltd.) and 10 pmol of each primer in a Biometra TPersonal Thermocycler (Biometra GmbH, Goettingen, Germany). The thermocycling conditions were as follows: Initial denaturation at $94^{\circ} \mathrm{C}$ for $3 \mathrm{~min}$, and three-step PCR for 30 cycles (denaturation at $94^{\circ} \mathrm{C}$ for $30 \mathrm{sec}$, annealing for $30 \mathrm{sec}$ at the appropriate annealing temperature depending on the primer $\left(51^{\circ} \mathrm{C}\right.$ for collagen I, $52^{\circ} \mathrm{C}$ for collagen III, $51^{\circ} \mathrm{C}$ for $\alpha$-SMA and $54^{\circ} \mathrm{C}$ for $\beta$-actin), and extension at $72^{\circ} \mathrm{C}$ for $60 \mathrm{sec}$ ), with a final extension at $72^{\circ} \mathrm{C}$ for $5 \mathrm{~min}$. The PCR products were combined and then separated by $1.0 \%$ agarose gels containing ethidium bromide (Sigma-Aldrich). Autoradiographic films of the RT-qPCR assays were subjected to densitometric analyses using a Kodak Gel Logic 100 (Kodak, Rochester, NY, USA). The PCR bands were analyzed using Quantity One 4.3.0 software (Bio-Rad Laboratories, Inc., Hercules, CA, USA). The amount of gene-specific PCR products was expressed as the ratio of the intensity of each band to that of the corresponding $\beta$-actin internal reference.

Detection of the protein expression levels of collagen I, collagen III and $\alpha$-SMA in the cell culture supernatant by ELISA. After cultivation for 3, 6, 9, 12, 15 and 18 days, the levels of collagen I and collagen III in the cell culture supernatant were detected by ELISA, whereas the levels of $\alpha$-SMA were detected after culture for 13, 16, 19, 22, 25 and 28 days. Collagen I, collagen III and $\alpha$-SMA in the cFbs culture supernatant were detected using a commercially available ELISA kit (Yixing Qianchen Bioengineering Company, Shanghai, China) according to the manufacturer's instructions.

Statistical analysis. All values are expressed as the mean \pm SD. SPSS 12.0 (SPSS Inc., Chicago, IL, USA) was used for statistical analysis. Statistical analysis of the differences between treated and untreated groups was performed by one-way analysis of variance, and by Dunnett's test for multigroup comparisons. $\mathrm{P}<0.05$ was considered to indicate a statistically significant difference. Graphic representation was performed using GraphPad Prism 5.0 for Windows (GraphPad Software, Inc., La Jolla, CA, USA).

\section{Results}

$\mathrm{SiO}_{2}$ inhibits the growth of rat AMs and cFbs. The primary culture model of rat AMs and cFbs in vitro is shown in Fig. 1A. Following treatment of macrophages with various concentrations of $\mathrm{SiO}_{2}(0,20,40,60,80,100$ and $120 \mu \mathrm{g} / \mathrm{ml})$ for $24 \mathrm{~h}$, the cell viability was detected using an MTT assay. As shown in Fig. 1B, compared with the control group $\left(0 \mu \mathrm{g} / \mathrm{ml} \mathrm{SiO}_{2}\right)$, treatment with $\mathrm{SiO}_{2}$ at concentrations of 100 and $120 \mu \mathrm{g} / \mathrm{ml}$ significantly inhibited the growth of AMs, with a significant decrease in cellular activity $(\mathrm{P}<0.05)$. To 
$\mathbf{A}$
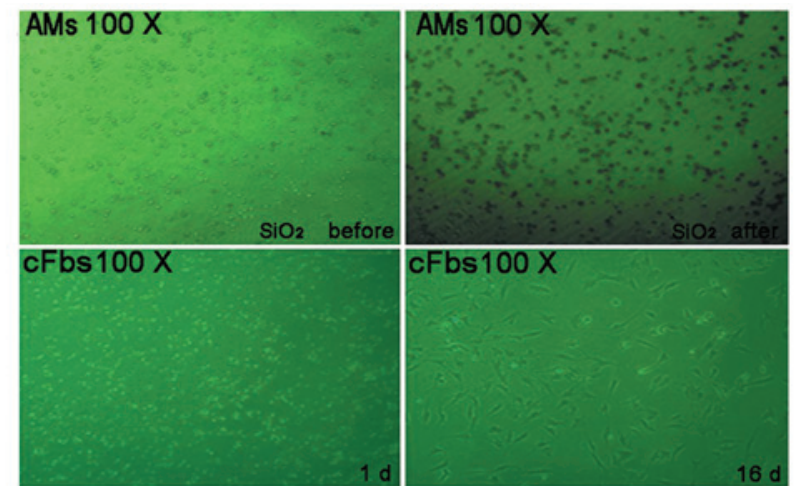

B

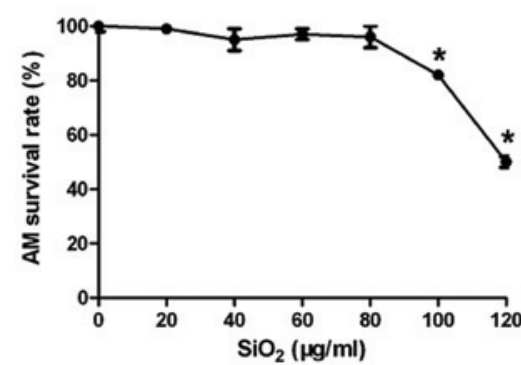

C

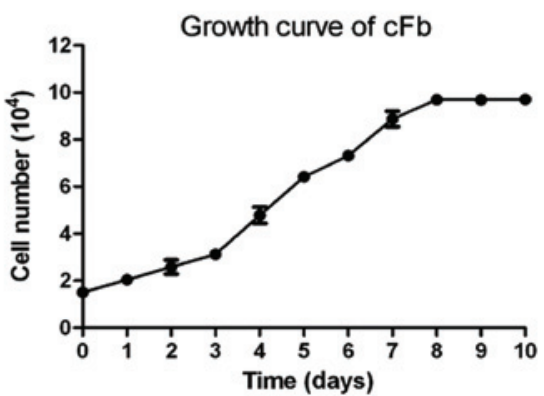

Figure 1. (A) Microscopic images of macrophages treated with $\mathrm{SiO}_{2}$ for $24 \mathrm{~h}$ and of cFbs treated with macrophage supernatant. (B) Growth curve of $\mathrm{cFbs}$. The survival rate of macrophages stimulated with $\mathrm{SiO}_{2}$ at $0,20,40,60,80,100$ or $120 \mu \mathrm{g} / \mathrm{ml}$ ( $\mathrm{n}=6$ per treatment group) as determined by MTT assay. (C) Assessment the effects of $\mathrm{SiO}_{2}$ on the proliferative rate of the cFbs. The cFbs were seeded and cultured with $80 \mu \mathrm{g} / \mathrm{ml} \mathrm{SiO}$ treated $\mathrm{AM}$ supernatant for $1,2,3,4,5,6,7,8,9$ or 10 days. Viable cells were detected by MTT assay and the cells were counted. Values are expressed as the mean \pm standard deviation. ${ }^{*} \mathrm{P}<0.05$, vs. the control group $\left(0 \mu \mathrm{g} / \mathrm{ml} \mathrm{SiO}_{2}\right.$-treated group). cFbs, circulating fibrocytes; $\mathrm{AMs}$, alveolar macrophages; $\mathrm{SiO}_{2}$, crystalline silica.

A

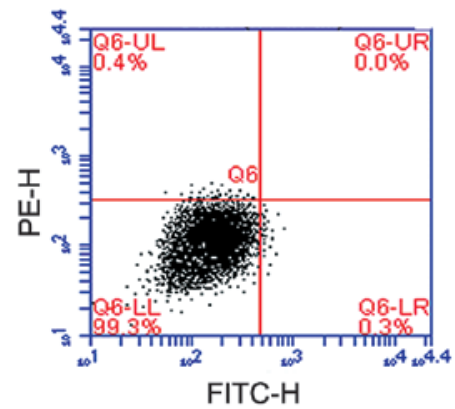

B

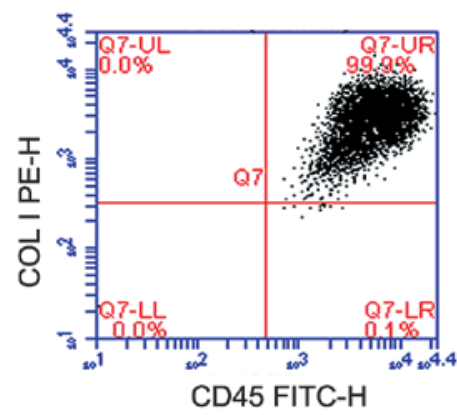

Figure 2. cFbs were identified by flow cytometry after 6 weeks of culture. Representative flow cytometric dot plots of circulating fibrocytes. (A) Isotype control for collagen 1 and CD45 using unstained cells. (B) Positive population for collagen 1 and CD45. COLI, collagen I; PE, phycoerythrin; FITC, fluorescein isothiocyanate; $\mathrm{cFbs}$, circulating fibrocytes.

further confirm the effects of $\mathrm{SiO}_{2}$ on the proliferative rate of $\mathrm{cFbs}$, the cFbs were cultured with AM supernatant, treated with $80 \mu \mathrm{g} / \mathrm{ml} \mathrm{SiO}_{2}$ and subjected to an MTT assay at 1 day intervals. The cFbs were seeded and cultured for 1-10 days, and harvested and counted each day. As shown in the growth curve (Fig. 1C), the doubling time of the cells was $96 \mathrm{~h}$, and the proliferative activity of the cFbs reached a peak between the fourth and eighth day.

Characterization of $c F b s$. cFbs were identified by flow cytometry, and the purity of the cFbs was assayed by staining with collagen I and CD45 antibodies after six weeks of culture. The results demonstrated that $99 \%$ of the cultured cells co-expressed collagen I and CD45 (Fig. 2).
$\mathrm{SiO}_{2}$ stimulates the protein expression of collagen I, collagen III and $\alpha-S M A$ in $c F b$ s. The present study investigated the effects of $\mathrm{SiO}_{2}$ on collagen I, collagen III and $\alpha$-SMA protein expression at various concentrations and incubation times by immunohistochemistry. As shown in Fig. 3A, collagen I, collagen III and $\alpha$-SMA was significantly increased in the cFbs following treatment with the supernatant of AM treated with $\mathrm{SiO}_{2}(0,20,40,60$ and $80 \mu \mathrm{g} / \mathrm{ml})$ for $24 \mathrm{~h}$. A dose-dependent increase in the expression levels of collagen I, collagen III and $\alpha$-SMA was observed in the cFbs treated with the supernatant of AM treated with 20,40 and $60 \mu \mathrm{g} / \mathrm{ml} \mathrm{SiO}_{2}$, as compared with that in the control group $(\mathrm{n}=6 ; \mathrm{P}<0.05)$, whereas in the $80 \mu \mathrm{g} / \mathrm{ml} \mathrm{SiO}_{2}$ group, the expression of the respective proteins was similar to that in the $60 \mu \mathrm{g} / \mathrm{ml} \mathrm{SiO}_{2}$ 
A

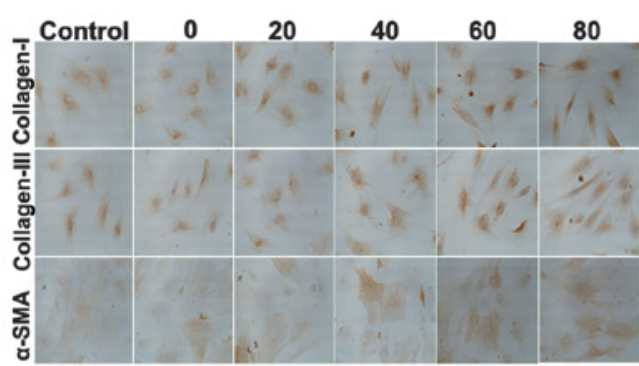

B

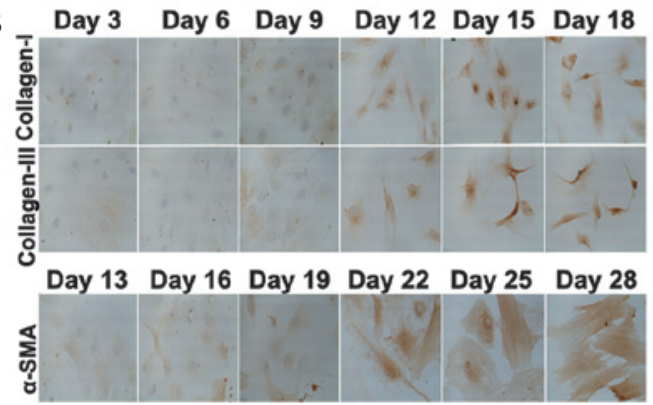

D
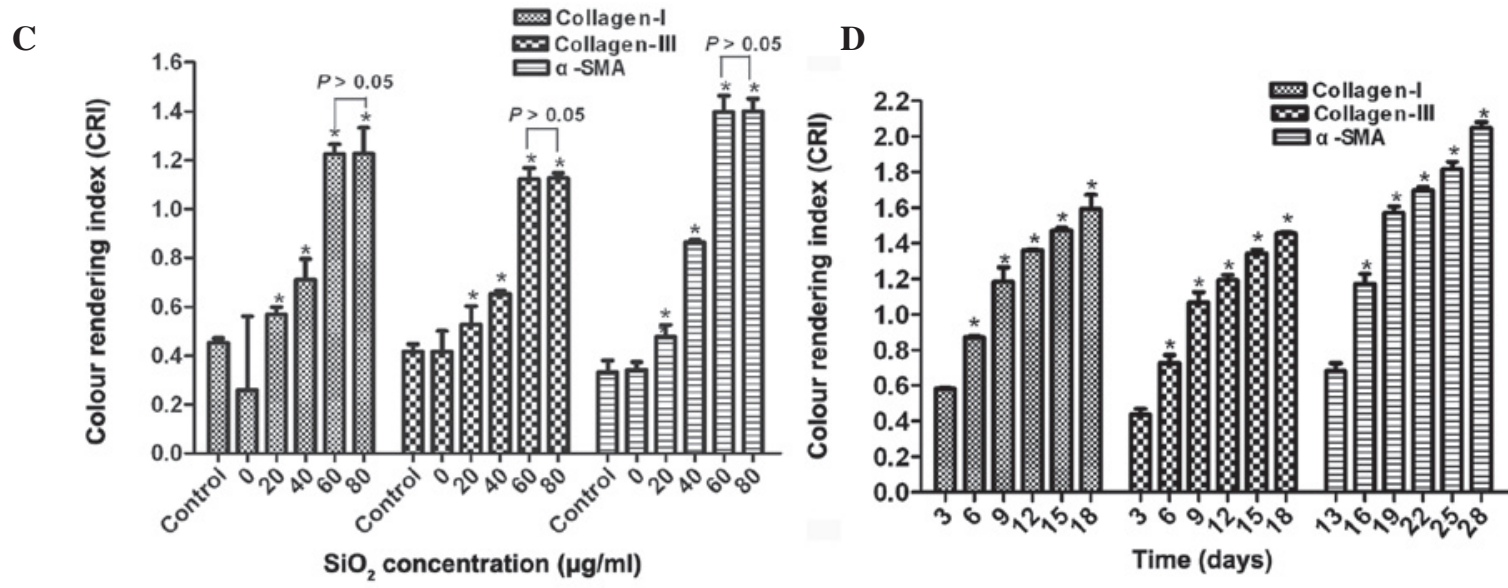

Figure 3. (A and B) Expression levels of collagen I, collagen III and $\alpha$-SMA in cFbs treated with the supernatant of alveolar macrophages treated with various concentrations of $\mathrm{SiO}_{2}$ for $24 \mathrm{~h}$, as determined by immunofluorescence (magnification, x100). (C and D) The staining intensity of collagen I, collagen III and $\alpha$-SMA was quantified by densitometric analysis. Values are expressed as the mean \pm standard deviation of three independent experiments $(\mathrm{n}=6)$. $" \mathrm{P}<0.05$, vs. the control group. cFbs, circulating fibrocytes; $\alpha$-SMA, $\alpha$ smooth muscle actin; $\mathrm{SiO}_{2}$, crystalline silica.

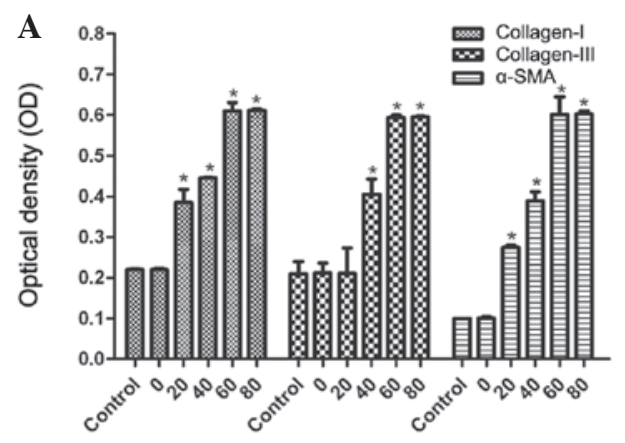

$\mathrm{SiO}_{2}$ concentration $(\mu \mathrm{g} / \mathrm{ml})$

C

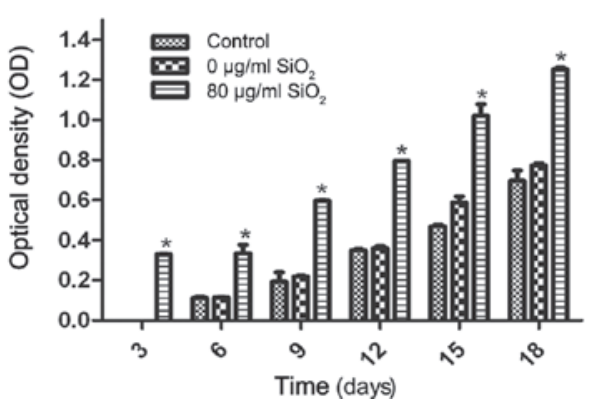

B

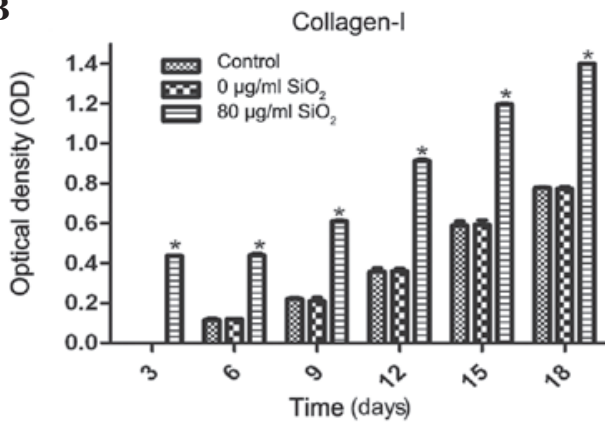

D

a-SMA

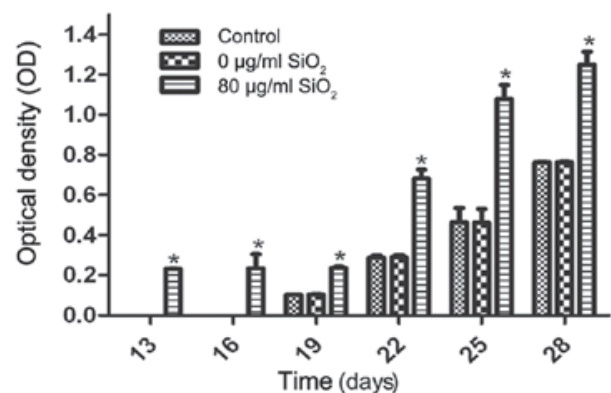

Figure 4. Protein expression levels of collagen I, collagen III and $\alpha$-SMA in cFbs, as determined by ELISA. (A) The cFbs were treated with the supernatant of AM treated with $\mathrm{SiO}_{2}(0,20,40,60$ and $80 \mu \mathrm{g} / \mathrm{ml})$ for $24 \mathrm{~h}$, and untreated cells served as the control group. The protein expression levels of collagen I, collagen III and $\alpha$-SMA were quantified and normalized relative to the control group. The bands were quantified by densitometric analysis. (B-D) Expression levels of collagen I, collagen III and $\alpha$-SMA following treatment with AM supernatant $\left(0\right.$ and $\left.80 \mu \mathrm{g} / \mathrm{ml} \mathrm{SiO}_{2}\right)$ for 3, 6, $9,12,15$ and 18 days for collagen I and collagen III, and for 13, 16, 19, 22, 25 and 28 days for $\alpha$-SMA. The cFb supernatant was collected and protein expression was determined by ELISA The expression levels of collagen I and collagen III were quantified and normalized relative to the control group. The bands were quantified by densitometric analysis. Values are expressed as the mean \pm standard deviation from three independent experiments $(\mathrm{n}=6)$. ${ }^{*} \mathrm{P}<0.01$, vs. the control. cFbs, circulating fibrocytes; $\alpha$-SMA, $\alpha$ smooth muscle actin; $\mathrm{SiO}_{2}$, crystalline silica; AM, alveolar macrophages. 
A

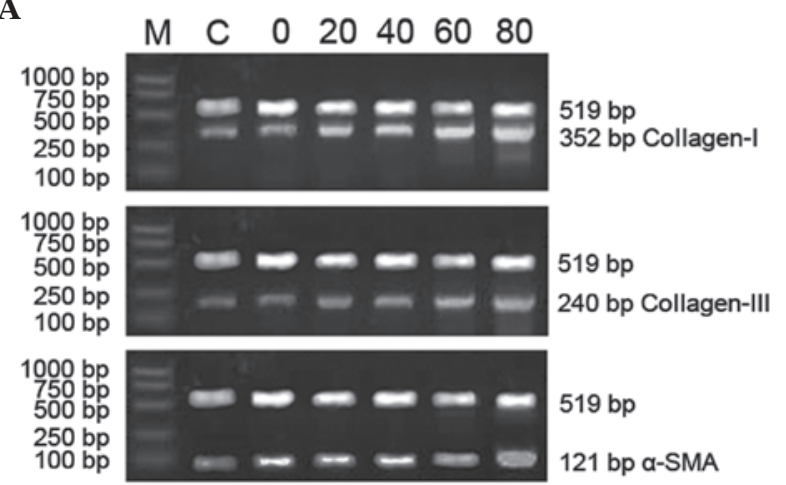

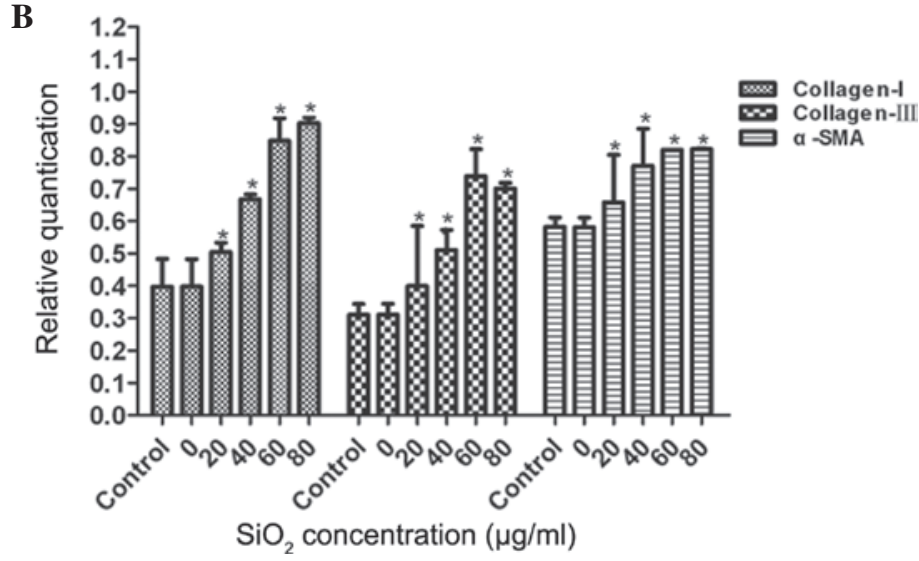

Figure 5. mRNA expression levels of collagen I, collagen III and $\alpha$-SMA in cFbs treated with the supernatant of AM treated with various concentrations of $\mathrm{SiO}_{2}$. The cFbs were treated with the supernatant of AM treated with $\mathrm{SiO}_{2}$ at concentrations of $0,20,40,60$ or $80 \mu \mathrm{g} / \mathrm{ml}$ for $24 \mathrm{~h}$, and the control group was not treated. (A) The cFbs were harvested, total RNA was extracted and the mRNA expression levels were determined by reverse transcription-quantitative polymerase chain reaction. (B) The mRNA expression levels of collagen I, collagen III and $\alpha$-SMA were quantified and normalized relative to internal $\beta$-actin mRNA. The bands were quantified by densitometric analysis. Values are expressed as the mean \pm standard deviation from three independent experiments $(\mathrm{n}=6) .{ }^{*} \mathrm{P}<0.01$, vs. the control group. cFbs, circulating fibrocytes; $\alpha$-SMA, $\alpha$ smooth muscle actin; $\mathrm{SiO}_{2}$, crystalline silica; AM, alveolar macrophages.

group ( $\mathrm{P}>0.05$; Fig. 3C). Fig. 3B shows representative staining for collagen I, collagen III and $\alpha$-SMA in cFbs following treatment for various durations with the supernatant of AM treated with $80 \mu \mathrm{g} / \mathrm{ml} \mathrm{SiO}_{2}$. The protein expression levels of collagen I, collagen III began to increase on day 3, and those of $\alpha$-SMA on day 13. As compared with the control group, the expression levels of collagen I and collagen III were significantly upregulated on days $6,9,12,15$ and $18(\mathrm{P}<0.05)$, whereas the expression levels of $\alpha$-SMA were significantly upregulated on days 16, 19, 22, 25 and 28 (P<0.05; Fig. 3D). These results suggested that $\mathrm{SiO}_{2}$ stimulated the expression levels of collagen I, collagen III and $\alpha$-SMA in a dose- and time-dependent manner.

In a further experiment, an ELISA was used to investigate the effects of $\mathrm{SiO}_{2}$ on the protein expression levels of collagen I, collagen III and $\alpha$-SMA in the cFbs cultured with the supernatant of AM treated with various concentrations of $\mathrm{SiO}_{2}$ for various durations. The cFbs were treated with AM supernatant treated with $\mathrm{SiO}_{2}$ at concentrations of 0,20 , 40,60 and $80 \mu \mathrm{g} / \mathrm{ml}$ for $24 \mathrm{~h}$, and untreated cFbs served as a control group. The protein expression levels of collagen I, collagen III and $\alpha$-SMA were quantified and normalized relative to the control group. As shown in Fig. 4A, a marked increase was observed in collagen I, collagen III and $\alpha$-SMA expression in the cFbs treated with $\mathrm{SiO}_{2}$ at concentrations of $20,40,60$ and $80 \mu \mathrm{g} / \mathrm{ml}$ for $24 \mathrm{~h}$, as compared with that in the control group. No statistically significant difference was observed between the $60 \mu \mathrm{g} / \mathrm{ml}$ and $80 \mu \mathrm{g} / \mathrm{ml}$ treatment groups $(\mathrm{P}>0.05 ; \mathrm{n}=6)$. Following $\mathrm{cFbs}$ treatment with $80 \mu \mathrm{g} / \mathrm{ml} \mathrm{SiO}_{2}$ for various durations, the protein expression levels of collagen I and collagen III began to increase on day 3 , and those of $\alpha$-SMA on day 13 , whereas the expression levels of collagen I and collagen III in the $0 \mu \mathrm{g} / \mathrm{ml} \mathrm{SiO}_{2}$ and the control groups began to increase on day 6 , and those of $\alpha$-SMA on day 19. In the treatment group, the expression levels of collagen I and collagen III were significantly upregulated on days $3,6,9,12,15$ and 18 , as compared with those in the control group ( $\mathrm{P}<0.05$; Fig. $4 \mathrm{~B}$ and $\mathrm{C})$, whereas the expression of $\alpha$-SMA was significantly upregulated on days 13, 16, 19, 22, 25 and 28 ( $\mathrm{P}<0.05$; Fig. 4D). These results suggested that $\mathrm{SiO}_{2}$ stimulated collagen I, collagen III and $\alpha$-SMA expression at the protein level and accelerated the processes of transdifferentiation of cFbs into myofibroblasts in a dose-dependent manner.

$\mathrm{SiO}_{2}$ stimulates the mRNA expression of collagen I, collagen III and $\alpha$-SMA in cFbs. To evaluate the effects of $\mathrm{SiO}_{2}$ on collagen I, collagen III and $\alpha$-SMA mRNA expression in $\mathrm{cFbs}$, the $\mathrm{cFbs}$ were treated with the supernatant of $\mathrm{SiO}_{2}$-treated AM $(20,40,60$ and $80 \mu \mathrm{g} / \mathrm{ml}$ for $24 \mathrm{~h})$. Treatment with the supernatant of AM treated with various concentrations of $\mathrm{SiO}_{2}$ increased the mRNA expression levels of collagen I, collagen III and $\alpha$-SMA in the cFbs. This increase in expression levels became apparent at $20 \mu \mathrm{g} / \mathrm{ml}$ $\mathrm{SiO}_{2}$ and markedly apparent at $80 \mu \mathrm{g} / \mathrm{ml} \mathrm{SiO}{ }_{2}$. The expression of collagen I, collagen III and $\alpha$-SMA was significantly and dose-dependently upregulated following treatment with 20 , 40 and $60 \mu \mathrm{g} / \mathrm{ml} \mathrm{SiO}_{2}$, as compared with that in the control group $(\mathrm{P}<0.05)$, while no statistically significant difference was observed between the $60 \mu \mathrm{g} / \mathrm{ml}$ and $80 \mu \mathrm{g} / \mathrm{ml}$ groups (P $>0.05$; Fig. 5A and B). Following treatment of the cFbs with the supernatant of $\mathrm{AM}$ treated with $80 \mu \mathrm{g} / \mathrm{ml} \mathrm{SiO}{ }_{2}$ for 28 days, the mRNA expression levels of collagen I, collagen III and $\alpha$-SMA were determined by RT-qPCR. As shown in Fig. 6, increases in collagen I and collagen III expression were detected on day three, and increases in $\alpha$-SMA expression was observed on day 13 of incubation, whereas in the control and $0 \mu \mathrm{g} / \mathrm{ml}$ groups, elevated collagen I and collagen III expression was detected on day 6 , and elevated $\alpha$-SMA expression on day 19. A significant upregulation of the expression levels of collagen I and collagen III in the treated groups was observed on days 3, 6, 9, 12, 15 and 18 $(\mathrm{P}<0.05)$. The expression levels of $\alpha$-SMA were significantly upregulated on days $13,16,19,22,25,28$, as compared with those in the control group $(\mathrm{P}<0.05)$. These results suggested that $\mathrm{SiO}_{2}$ was able to stimulate collagen I, collagen III and $\alpha$-SMA activity at the transcriptional level, and increase the mRNA synthesis of collagen I, collagen III and $\alpha$-SMA. 
A

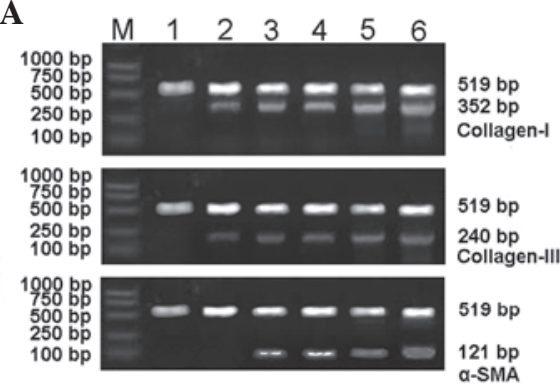

B

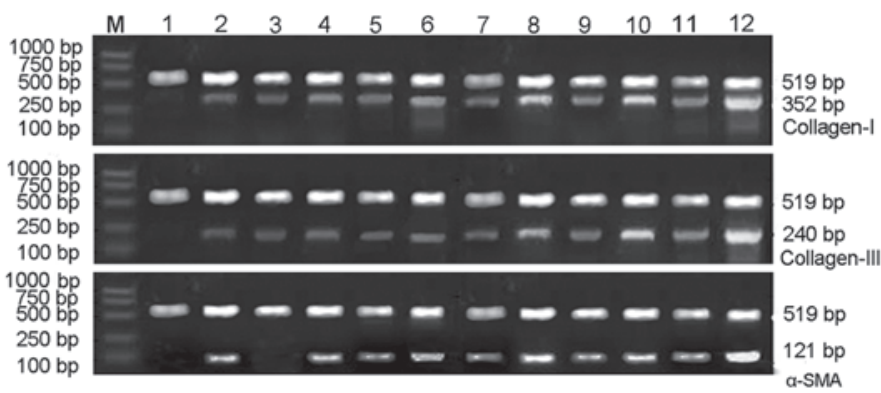

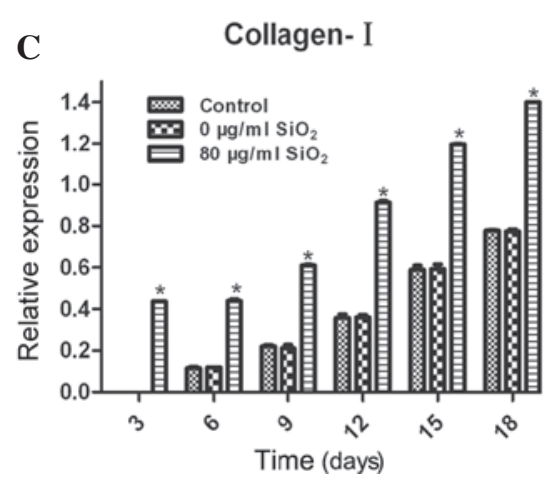

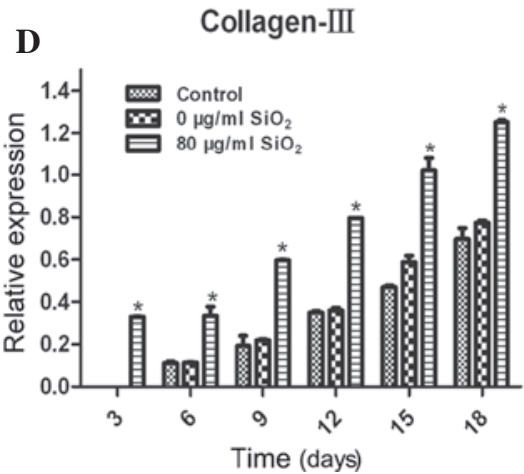

$\mathbf{E}$

a-SMA

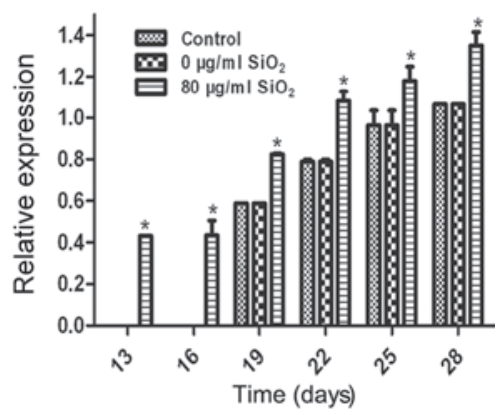

Figure 6. mRNA expression levels of collagen I, collagen III and $\alpha$-SMA in cFbs. The cFbs were treated with alveolar macrophage supernatant treated with $\mathrm{SiO}_{2}$ at concentrations of 0 and $80 \mu \mathrm{g} / \mathrm{ml}$ for $24 \mathrm{~h}$, and untreated cells served as the control group. (A) Collagen I and collagen III detection was performed following cultivation with AM supernatant $\left(0 \mu \mathrm{g} / \mathrm{ml} \mathrm{SiO}_{2}\right)$ for (1) 3, (2) 6, (3) 9, (4) 12, (5) 15 and (6) 18 days, and $\alpha$-SMA detection was performed following cultivation for (1) 13, (2) 16, (3) 19, (4) 22, (5) 25 and (6) 28 days. (B) Collagen I and collagen III detection was performed following treatment with AM supernatant $\left(80 \mu \mathrm{g} / \mathrm{ml} \mathrm{SiO}_{2}\right)$ for (1) 3, (2) 6, (3) 9, (4) 12, (5) 15 and (6) 18 days, and $\alpha$-SMA detection was performed following cultivation for (1) 13, (2) 16, (3) 19, (4) 22 , (5) 25 and (6) 28 days. Total RNA was extracted and quantified by reverse transcription-quantitative polymerase chain reaction. The mRNA expression levels of collagen I, collagen III and $\alpha$-SMA were quantified and normalized relative to the mRNA expression levels of $\beta$-actin. (C-E) The bands were quantified by densitometric analysis and are shown in histograms. Values are expressed as the mean \pm standard deviation from three independent experiments ( $\mathrm{n}=6$ ). ${ }^{*} \mathrm{P}<0.01$, vs. the control group. cFbs, circulating fibrocytes; $\alpha$-SMA, $\alpha$ smooth muscle actin; $\mathrm{SiO}_{2}$, crystalline silica.

\section{Discussion}

The present study demonstrated that $\mathrm{SiO}_{2}$ treatment accelerated $\mathrm{cFb}$ differentiation in a concentration- and time-dependent manner. In patients with ongoing lung fibrosis, blood monocytes may penetrate into the alveolar space, where they differentiate into fibrocytes (21). cFbs have previously been reported to be increased in patients with idiopathic pulmonary fibrosis (7). Silicosis is characterized by persistent inflammation, leading to fibroblast formation and excessive collagen deposition, which causes interstitial fibrosis and silicotic nodule formation (22). The presence of $\mathrm{cFbs}$ may serve as a prognostic biomarker for silicosis.

In healthy individuals, fibrocytes comprise $0.1-1.0 \%$ of the peripheral blood nucleated cells, and are present in a variety of healthy as well as diseased tissue types (5). Fibrocytes from human peripheral blood are circulating progenitor cells with the ability to differentiate into osteoblasts and chondrocyte-like cells (23). These cells remain active during tissue remodeling through the elaboration of extracellular matrix (ECM) proteins (collagen I and collagen III) and secrete various inflammatory mediators (cytokines, chemokines and growth factors) (5). Of note, fibrocytes deposit ECM components during various fibroproliferative disorders of the lung (14). The present study aimed to investigate differentiated fibrocytic cell lines expressing the myofibroblast marker $\alpha$-SMA. The present study observed changes in collagen I, collagen III and $\alpha$-SMA expression associated with the development of $\mathrm{cFbs}$ treated with $\mathrm{SiO}_{2}$. The proliferative activity levels of AMs treated with $\mathrm{SiO}_{2}$ were assessed using an MTT assay and the effects of their supernatants on cFbs were investigated by observing the mRNA and protein expression levels of collagen I, collagen III and $\alpha$-SMA over the course of 28 days. A previous study reported that there are three stages in the process of cFbs cultivation in vitro: An early developmental stage over the course of the first three days, a differentiation stage from the fourth to the sixth day, and a ripening stage from the seventh day to the 18th day (17). The results of the present study demonstrated that the expression levels of collagen I, collagen III and $\alpha$-SMA in cFbs increased at different time-points: Collagen I and collagen III expression increased on day 3 , while $\alpha$-SMA expression increased on day 13, and these expression levels continued to increase on days 3, 5, 7, 9 and 12 for collagen I and collagen III, and on days 13, 15, 17, 19 and 23 for $\alpha$-SMA.

The mRNA expression levels of collagen I, collagen III and $\alpha$-SMA in the experimental groups were significantly higher as compared with those in the control group, and the expression levels were increased by $\mathrm{SiO}_{2}$ treatment in a dose-dependent manner. No statistically significant difference was observed between the $0 \mu \mathrm{g} / \mathrm{ml} \mathrm{SiO}_{2}$ treatment group and the control group $(\mathrm{P}>0.05)$. Therefore, the mRNA expression levels of collagen I, collagen III and $\alpha$-SMA were increased 
by $\mathrm{SiO}_{2}$ in a dose-dependent manner. As compared with the control group, marked increases were observed in the mRNA expression levels of collagen I, collagen III and $\alpha$-SMA in the treated groups. These results indicated that $\mathrm{SiO}_{2}$ promoted the transdifferentiation of cFbs into myofibroblasts, as deduced from the marked increment of $\alpha$-SMA expression. Increases in the mRNA expression levels of collagen I and collagen III were detected on day 6 , those of $\alpha$-SMA were detected on day 19, and mRNA levels continued to increase in a time-dependent manner.

The majority of liver injuries feature collagen accumulation and the destruction of normal liver architecture by fibrosis, a process that involves activated myofibroblasts that arose from local fibroblast precursors (24). $\alpha$-SMA is expressed on the surface of fibrocytes and is a surface marker of myofibroblasts $(7,8)$. As compared with the control group, following treatment with AM supernatant treated with $\mathrm{SiO}_{2}$ at concentrations of $0,20,40,60$ and $80 \mu \mathrm{g} / \mathrm{ml}$ for $24 \mathrm{~h}$, a marked increase in the expression levels of collagen I, collagen III and $\alpha$-SMA was observed in the cFbs, as determined by immunohistochemistry and ELISA. No statistically significant difference was observed between the $60 \mu \mathrm{g} / \mathrm{ml}$ and $80 \mu \mathrm{g} / \mathrm{ml}$ treatment groups. These results suggested that $80 \mu \mathrm{g} / \mathrm{ml} \mathrm{SiO}$ may exhibit toxicity to $\mathrm{cFbs}$, inhibiting the growth of $\mathrm{cFbs}$. As determined by immunohistochemistry, following $\mathrm{cFb}$ treatment with $80 \mu \mathrm{g} / \mathrm{ml} \mathrm{SiO}_{2}$ for various durations, the expression levels in the $80 \mu \mathrm{g} / \mathrm{ml}$ treatment group were markedly elevated as compared with those in the control group, and these expression levels increased in a dose-dependent manner with increasing $\mathrm{SiO}_{2}$ concentration $(\mathrm{P}<0.05)$. In the $0 \mu \mathrm{g} / \mathrm{ml}$ treatment and the control group, the protein expression levels of collagen I and collagen III were markedly increased on day 3 , whereas those of $\alpha$-SMA were markedly increased on day 16, as determined by ELISA. The protein expression levels of collagen I and collagen III in the $0 \mathrm{mg} / \mathrm{kg}$ treatment and the control groups markedly increased on day 6 , whereas those of $\alpha$-SMA increased on day 19 . The variation in the results obtained by immunohistochemical analysis and ELISA indicated that collagen I, collagen III and $\alpha$-SMA were expressed earlier in the cells, as compared with their presence in the culture supernatant. These results suggested that $\mathrm{SiO}_{2}$ was able to stimulate translational expression of collagen I, collagen III and $\alpha$-SMA and stimulate $\mathrm{cFb}$ transformation in a dose-dependent manner and the protein expressions of $\alpha$-SMA, collagen I and collagen III in cells were similar to that of 3 days earlier in the culture supernatant at the same concentration of $\mathrm{SiO}_{2}$.

The expression levels of collagen I and collagen III observed in the cFbs treated with $80 \mu \mathrm{g} / \mathrm{ml} \mathrm{SiO}_{2}$ were significantly higher, as compared with those in the $0 \mu \mathrm{g} / \mathrm{ml}$ treatment or control groups. Similar cellular distribution and appearance was observed in cells expressing $\alpha$-SMA, as demonstrated by immunohistochemical analysis of cFbs. These results indicated upon treatment with the supernatant of AM treated with $\mathrm{SiO}_{2}, \mathrm{cFbs}$ produced more collagen I, collagen III and $\alpha$-SMA. It has been proposed that fibrocytes may be a transitional stage between a monocyte and a fibroblast (25). Previous studies have demonstrated the role of myofibroblasts in pulmonary fibrotic processes (26), and cFbs are capable of differentiating into myofibroblasts in vitro and in vivo (27). Concordant with these previous observations, in the present study, the presence of significantly higher levels of $\alpha$-SMA expression by fibrocytes treated with the supernatant of AM treated with $\mathrm{SiO}_{2}$, as compared with those in the control group (untreated), suggested and important role for $\alpha$-SMA in the transformation of cFbs. The observations of the present study suggested that the time required for leukocytes to differentiate into cFbs was accelerated by $\mathrm{SiO}_{2}$ treatment in a concentration-dependent manner.

The present study provided numerous important implications which serve as a basis for future studies. Considering that the silicosis-induced fibrotic process is similar to that of other fibrotic pulmonary diseases, the fibroproliferative effects of $\mathrm{SiO}_{2}$ on cFbs are of paramount importance. In addition, the effects of cFbs in the development and progression of silicosis prompt further investigation. Future studies investigating the mechanisms underlying the role of $\mathrm{cFbs}$ in silicosis in the human body would be of great value.

Despite the significant aforementioned results, the present study has notable limitations. It remains elusive whether cFbs differentiate into myofibroblasts during the development and progression of silicosis in vivo. As the present study only provided results based on in vitro findings, future studies are required to further characterize the changes in cFbs that occur during the development and progression of silicosis. However, the present study represents an important contribution to scientific research and indicated that $\mathrm{cFbs}$ may represent a novel therapeutic target in the treatment of silicosis.

In conclusion, the present study provided a novel therapeutic approach, and determined that the overexpression of collagen I, collagen III and $\alpha$-SMA protein as well as mRNA in $\mathrm{cFbs}$ in the peripheral blood may have an important role in the development of silicosis. In addition, the results of the present study may provide novel methods of silicosis prevention and control. The present study investigated the effects of $\mathrm{SiO}_{2}$ on collagen I, collagen III and $\alpha$-SMA protein and mRNA in cFbs in vitro. To the best of our knowledge, the present study was the first to demonstrate that the transdifferentiation of $\mathrm{cFbs}$ into myofibroblasts was accelerated by $\mathrm{SiO}_{2}$. cFbs may represent a novel therapeutic target for developing more effective treatments against silicosis

\section{Acknowledgements}

The authors of the present study are grateful to the staff of the Department of Public Health of the Zhengzhou University. The present study was supported by a grant from the National Natural Science Foundation of China (grant. no. 81102109).

\section{References}

1. Athavale A, Iyer A, Sahoo D, Salgia K, Raut A and Kanodra N: Incidence of silicosis in flourmill workers. Indian J Occup Environ Med 15: 104-108, 2011.

2. Sharawy MH, El-Agamy DS, Shalaby AA and Ammar el-SM: Protective effects of methyl palmitate against silica-induced pulmonary fibrosis in rats. Int Immunopharmacol 16: 191-198, 2013.

3. Ekert JE, Murray LA, Das AM, Sheng H, Giles-Komar J and Rycyzyn MA: Chemokine (C-C motif) ligand 2 mediates direct and indirect fibrotic responses in human and murine cultured fibrocytes. Fibrogenesis Tissue Repair 4: 23, 2011. 
4. Bucala R, Spiegel LA, Chesney J, Hogan M and Cerami A Circulating fibrocytes define a new leukocyte subpopulation that mediates tissue repair. Mol Med 1: 71-81, 1994.

5. Quan TE, Cowper S, Wu SP, Bockenstedt LK and Bucala R: Circulating fibrocytes: Collagen-secreting cells of the peripheral blood. Int J Biochem Cell Biol 36: 598-606, 2004.

6. Xia D,MoyanaT and Xiang J: Combinational adenovirus-mediated gene therapy and dendritic cell vaccine in combating well-established tumors. Cell Res 16: 241-259, 2006.

7. Moeller A, Gilpin SE, Ask K, Cox G, Cook D, Gauldie J, Margetts PJ, Farkas L, Dobranowski J, Boylan C, et al: Circulating fibrocytes are an indicator of poor prognosis in idiopathic pulmonary fibrosis. Am J Respir Crit Care Med 179: 588-594, 2009

8. Abu El-Asrar AM, Struyf S, Van Damme J and Geboes K: Circulating fibrocytes contribute to the myofibroblast population in proliferative vitreoretinopathy epiretinal membranes. $\mathrm{Br} J$ Ophthalmol 92: 699-704, 2008.

9. Abe R, Donnelly SC, Peng T, Bucala R and Metz CN: Peripheral blood fibrocytes: Differentiation pathway and migration to wound sites. J Immunol 166: 7556-7562, 2001.

10. Moore BB, Murray L, Das A, Wilke CA, Herrygers AB and Toews GB: The role of CCL12 in the recruitment of fibrocytes and lung fibrosis. Am J Respir Cell Mol Biol 35: 175-181, 2006.

11. Abe S, Boyer C, Liu X, Wen FQ, Kobayashi T, Fang Q, Wang X, Hashimoto M, Sharp JG and Rennard SI: Cells derived from the circulation contribute to the repair of lung injury. Am J Respir Crit Care Med 170: 1158-1163, 2004.

12. Moore BB, Kolodsick JE, Thannickal VJ, Cooke K, Moore TA Hogaboam C, Wilke CA and Toews GB: CCR2-mediated recruitment of fibrocytes to the alveolar space after fibrotic injury. Am J Pathol 166: 675-684, 2005.

13. Murray LA, Argentieri RL, Farrell FX, Bracht M, Sheng H, Whitaker B, Beck H, Tsui P, Cochlin K, Evanoff HL, et al: Hyper-responsiveness of IPF/UIP fibroblasts: Interplay between TGFbeta1, IL-13 and CCL2. Int J Biochem Cell Biol 40 2174-2182, 2008.

14. Phillips RJ, Burdick MD, Hong K, Lutz MA, Murray LA, Xue YY, Belperio JA, Keane MP and Strieter RM: Circulating fibrocytes traffic to the lungs in response to CXCL12 and mediate fibrosis. J Clin Invest 114: 438-446, 2004.

15. Gomer RH: Circulating progenitor cells and scleroderma. Curr Rheumatol Rep 10: 183-188, 2008.
16. Hong KM, Belperio JA, Keane MP, Burdick MD and Strieter RM: Differentiation of human circulating fibrocytes as mediated by transforming growth factor-beta and peroxisome proliferator-activated receptor gamma. J Biol Chem 282: 22910-22920, 2007.

17. Hrckova G, Velebný S and Solár P: Dynamics of hepatic stellate cells, collagen types I and III synthesis and gene expression of selected cytokines during hepatic fibrogenesis following Mesocestoides vogae (Cestoda) infection in mice. Int J Parasitol 40: 163-174, 2010.

18. Joddar B and Ramamurthi A: Fragment size- and dose-specific effects of hyaluronan on matrix synthesis by vascular smooth muscle cells. Biomaterials 27: 2994-3004, 2006.

19. Yang Y, Li J, Mao S and Zhu H: Comparison of immunohistology using pan-CK and EMA in the diagnosis of lymph node metastasis of gastric cancer, particularly micrometastasis and isolated tumor cells. Oncol Lett 5: 768-772, 2013.

20. Feldmann G, Fendrich V, McGovern K, Bedja D, Bisht S, Alvarez H, Koorstra JB, Habbe N, Karikari C, Mullendore M, et al: An orally bioavailable small-molecule inhibitor of Hedgehog signaling inhibits tumor initiation and metastasis in pancreatic cancer. Mol Cancer Ther 7: 2725-2735, 2008.

21. Reese C, Lee R, Bonner M, Perry B, Heywood J, Silver RM, Tourkina E, Visconti RP and Hoffman S: Fibrocytes in the fibrotic lung: Altered phenotype detected by flow cytometry. Front Pharmacol 5: 141, 2014.

22. Thakur SA, Beamer CA, Migliaccio CT and Holian A: Critical role of MARCO in crystalline silica-induced pulmonary inflammation. Toxicol Sci 108: 462-471, 2009.

23. Choi YH, Burdick MD and Strieter RM: Human circulating fibrocytes have the capacity to differentiate osteoblasts and chondrocytes. Int J Biochem Cell Biol 42: 662-671, 2010.

24. Guyot C, Lepreux S, Combe C, Doudnikoff E, Bioulac-Sage P, Balabaud C and Desmoulière A: Hepatic fibrosis and cirrhosis: The (myo)fibroblastic cell subpopulations involved. Int J Biochem Cell Biol 38: 135-151, 2006.

25. Reilkoff RA, Bucala R and Herzog EL: Fibrocytes: Emerging effector cells in chronic inflammation. Nat Rev Immunol 11: 427-435, 2011.

26. Phan SH: The myofibroblast in pulmonary fibrosis. Chest 122 (Suppl 6): 286S-289S, 2002.

27. Schmidt M, Sun G, Stacey MA, Mori L and Mattoli S: Identification of circulating fibrocytes as precursors of bronchial myofibroblasts in asthma. J Immunol 171: 380-389, 2003. 